\title{
PCE: What is It, How Does It Work and What are its Limitations?
}

\author{
Raul Muñoz, Ramon Casellas, Ricardo Martínez and Ricard Vilalta
}

\begin{abstract}
In GMPLS-controlled optical networks, the utilization of source-based path computation has some limitations, especially in large networks with stringent constraints (e.g. optical impairments) or in multi-layer and multi-domain networks, which leads to sub-optimal routing solutions. The Path Computation Element (PCE) can mitigate some weaknesses of GMPLS-controlled optical networks. The main idea behind the PCE is to decouple the path computation function from the GMPLS controllers into a dedicated entity with an open and well-defined interface and protocol. A (stateless) PCE is capable of computing a network path or route based on a network graph (i.e., the Traffic Engineering Database - TED) and applying computational constraints. First, we present an overview of the PCE architecture and its communication protocol (PCEP). Then, we present in detail the considered source-routing shortcomings in GMPLS-controlled networks, namely, impairment-aware path computation, multi-domain path computation and multi-layer path computation, as well as the different PCE-based solutions that have been proposed to overcome each one of these problems. However, PCE-based computation also presents some limitations that lead to an increase in the path computation blocking or to sub-optimal path computations. The stateful PCE overcomes the limitations of the stateless PCE, such as the outdated TED, the lack of global LSP state (i.e., set of computed paths and reserved resources in use in the network), and the lack of control of path reservations. A passive stateful PCE allows optimal path computation and increased path computation success, considering both the network state (TED) and the Label Switched Paths (LSP) state (LSP Database - LSPDB). Additionally, an active stateful PCE can modify existing LSPs (i.e., connections), and optionally, setup and/or release existing LSPs. Finally, the formal decoupling of the path computation allows more flexibility in the deployment of PCEs in other control paradigms outside their original scope (MPLS/GMPLS). In this sense, we provide an overview of three PCE deployment models in the Software Defined Network (SDN) control architecture.
\end{abstract}

Index Terms-Path Computation Element (PCE), control plane, Generalized Multiprotocol Label Switching (GMPLS), Software Defined Networks (SDN), stateful PCE, stateless PCE, OpenFlow, multi-layer path computation, multi-domain path computation, optical networks.

\section{INTRODUCTION}

$\mathbf{T}$ TRADITIONALLY, the deployment of a Generalized Multi-Protocol Label Switching (GMPLS) [1] control plane architecture has encompassed the automation of optical network functionalities such as connection provisioning and recovery (i.e., protection and restoration), traffic engineering

This paper was supported by the European Community's Seventh Framework Programme FP7/2007-2013 under grant agreement n 317999 (IDEALIST project) and the Spanish Ministry of Economy and Competitiveness (MINECO) through the project FARO (TEC2012-38119).

R. Muñoz, R. Casellas, R. Martínez and R. Vilalta are with the Centre Tecnològic de Telecomunicacions de Catalunya (CTTC), Av. Carl Friedrich Gauss 7, 08860 Castelldefels (Barcelona), Spain, e-mail: \{raul.munoz, ramon.casellas, ricardo.martinez, ricard.vilalta \}@cttc.es or QoS [2]. The GMPLS architecture defines a set of standard protocols constituted by three pillars: a signaling protocol (i.e., RSVP-TE [3]) used for setting up the end-to-end connections (Label Switched Paths - LSPs), a routing protocol (i.e., OSPFTE [4]) used for topology and network resource dissemination, and a link management protocol (i.e., LMP [5]) for control channel connectivity maintenance, link property correlation, and fault localization.

A GMPLS control plane is a distributed entity composed of controllers (one per node) which execute several collaborative processes (Connection Controller -CC-, Routing Controller -RC-, path computation, etc.), and a data communication network based on IP control channels (IPCC) to allow the exchange of control messages between GMPLS controllers (Fig. 1.a). A GMPLS-enabled node (both control and hardware) is named Label Switched Router (LSR) in the GMPLS architecture. Under distributed control, each GMPLS controller manages the state of all the connections (i.e., LSPs) originated, terminated or passing-through a node, stored in the LSP Database (LSPDB), and maintains its own network state information (topology and resources), collected in a local TED repository. For each managed connection, the GMPLS controller is responsible for executing efficient/optimum path computation algorithms (i.e., distributed source routing/ path computation) to find a feasible end-to-end path based on the network state information.

The routing controller is responsible for disseminating changes occurring in the network state through the GMPLS OSPF-TE routing protocol, allowing the nodes' routing controllers to update their local TEDs and maintain a global picture of the current network topology and resource availability. After a network state change, the node originating the change generates and floods a Traffic Engineering - Link State Advertisement (TE LSA) to all its neighboring nodes (Fig. 1.b). The neighboring nodes receive the TE LSAs, forward them, and update their TED repositories. This mechanism allows synchronizing all the nodes' TED repositories (i.e., all node's routing controllers have the same and unique view of the network state within a given time, referred to as the routing convergence time). In GMPLS-controlled optical transport networks, it is possible to operate either on an aggregated basis (flooding information on the unreserved bandwidth/spectrum) or enabling the dissemination of the status of the wavelength channels in Wavelength Switched Optical Networks (WSON) or Nominal Central Frequencies (NCF) in Spectrum Switched Optical Networks (SSON) on a per TE link basis. SSON is also known as elastic optical Network or flexi-grid DWDM network.

With source-based path computation, upon reception of 


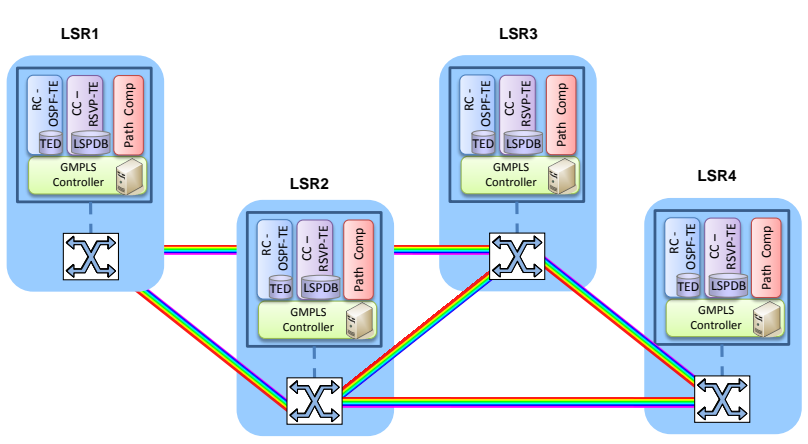

(a) Example of GPMLS-controlled optical network

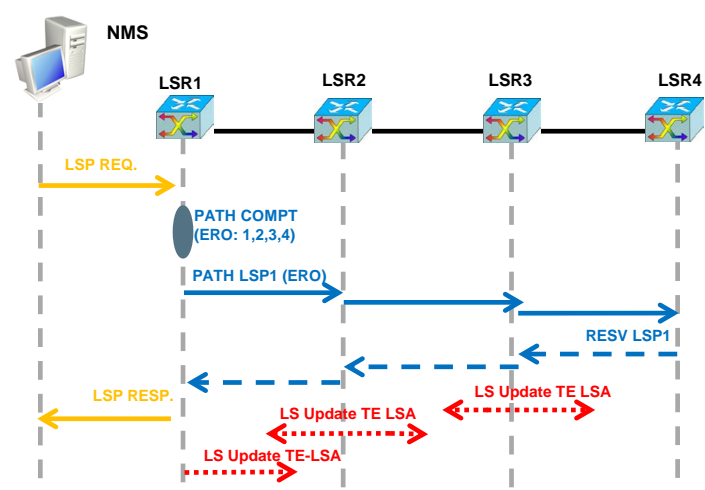

(b) Example of GMPLS source-based path computation and provisioning

Fig. 1: GMPLS-based distributed control plane

a connection request at the source node (e.g., through a Network Management System - NMS), such a node executes a path computation algorithm (e.g., routing and wavelength assignment - RWA - in WSON, or routing and spectrum assignment - RSA - in SSON) to find a feasible end-toend path. The computed path is passed to the connection controller as Explicit Route Objects (ERO). The subsequent GMPLS RSVP-TE signaling mechanism consists of a label request within a RSVP Path message from the source to the destination node (i.e., forward direction), and a generalized label assignment (reservation) sent in a RSVP Resv message which travels backwards to the source node (i.e., backward direction), as shown in Fig.1.b). This exchange of signaling messages allows each involved connection controller to update its LSPDB repository.

Dynamic path computation can be addressed considering a combined or a distributed approach. In a combined routing and resource assignment approach, the source node computes both the spatial path (nodes and links) and assigns the resources (wavelengths, frequency slot, 3R regenerators, wavelengthconverters, etc.) using as input the TED information. This approach requires that the routing protocol disseminates detailed network information (e.g., wavelength/NCF, 3R, wavelength converters, etc.). In a routing and distributed resource assignment approach, the source node only computes the spatial path using TED information (e.g., link aggregated unreserved bandwidth), but resource assignment is performed at the destination and intermediate nodes during signaling in the backwards direction. This approach requires collecting resource state information (e.g., wavelength/NCF, 3R regenerators, wavelength converters, etc.) during the signaling in the forward direction.

Future optical transport networks will be constituted by several interconnected administrative domains, each one supporting a single or multiple switching layers (e.g., packets, time slots, wavelengths or spectrum). In such a scenario, the utilization of source-based path computation is limited, especially in large networks with stringent constraints or in multilayer and multi-domain networks, which leads to sub-optimal routing solutions. First, this paper overviews in Sec.II, the Path Computation Element (PCE) architecture to overcome the source-based routing shortcomings. Then, we detail in Sec.III, the limitations of source path computation in the context of optical, multi-layer and multi-domain GMPLS networks and present how the PCE can overcome these weaknesses. After that, we identify in Sec.IV some of the limitations of the stateless PCE to evolve towards the stateful PCE in Sec.V, as the solution to the weaknesses of the stateless PCE. Finally, in Sec. VI we present PCE deployment models in Software Defined Networks (SDN) control architectures, and we conclude the paper in Sec.VII.

\section{BASICS OF THE PCE ARCHITECTURE}

A PCE is an entity (component, application or network node) that is capable of computing a network path or route based on a network graph (TED) and applying computational constraints. The main idea is to decouple the path computation function from the GMPLS controllers into centralized and dedicated PCE with an open and well-defined interface and protocol (Fig.2.a). The IETF defined a standard and functional formalization of the PCE global architecture and the communication interface and protocol (PCEP) in 2006 for MPLS path computation [6] and eventually extended for GMPLS [7]. By doing so, Path Computation Clients (PCCs) such as GMPLS Controllers may request the computation of an explicit route (Fig. 2.b).

The initial driver for the deployment of PCEs was the increasing complexity of path computation. In order to efficiently use the network resources, advanced path computation mechanisms are needed to assign concrete resources (e.g., bandwidth) to client connection requests, considering the diversity and variety of the received client connection requirements as well as the traffic engineering attributes of network elements (e.g., nodes and links). In the context of WSON/SSON, path computation cannot easily rely on constrained shortest path first (CSPF) approaches based on additive metrics with convex restrictions (e.g., bandwidth). Indeed, it needs to consider, as a bare minimum, discrete wavelength/NCF availability with potential wavelength or spectrum continuity constraints and optical impairments. Moreover, the optical technology may also imply restrictions regarding node internal connectivity, tunability/signal limitations, or the availability of $3 \mathrm{R}$ regenerator pools. Consequently, having this functional component replicated within each controller may increase its cost given the increasing computational complex- 


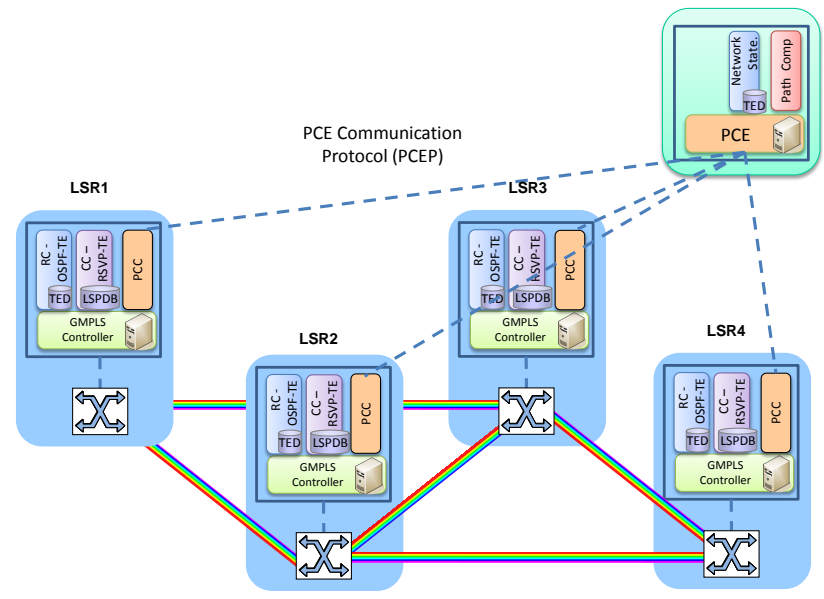

(a) Example of GPMLS-controlled optical network with stateless PCE

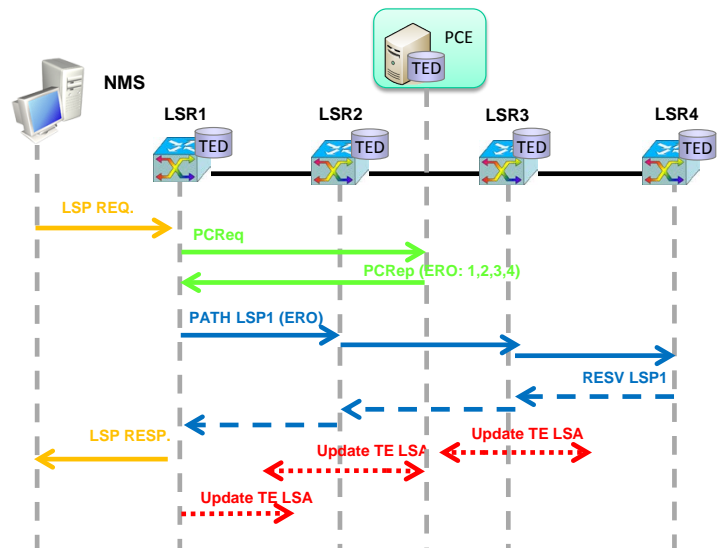

(b) Example of PCE path computation and GMPLS provisioning

Fig. 2: GMPLS-based distributed control plane with stateless PCE

ity, which often require ad-hoc algorithms and configurable policies and rules.

The decoupling of the path computation from the rest of the control plane also provides further benefits:

- More flexibility for network operators in the control of their networks

- The ability to apply network operators' policies in the development of the path computation algorithms, not bound to software updates within the controllers (closed and vendor-dependent)

- Third party customized developments and upgrades of path computation algorithms

As mentioned above, The PCE architecture has two main components, namely, the PCC (any client application requesting a path computation to a PCE), and a PCE. On the one hand, a PCC can be located within a network node (e.g. GMPLS controller) or in the NMS. A PCC may use different PCEs for path computation (e.g., to distribute the set of requests for load balancing purposes). On the other hand, the PCE entity is an application that can be located within a network node (composite PCE node) or in a dedicated server (external PCE node).

The PCE architecture supports two path computation mod-
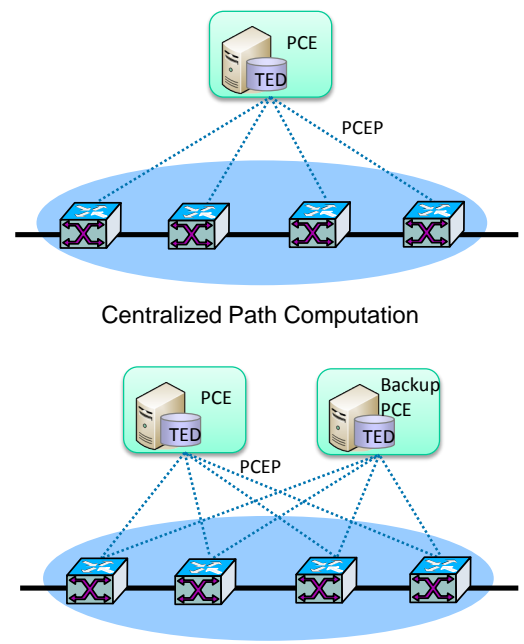

Centralized Path Computation with backup

(a) PCE-based Centralized Path Computation
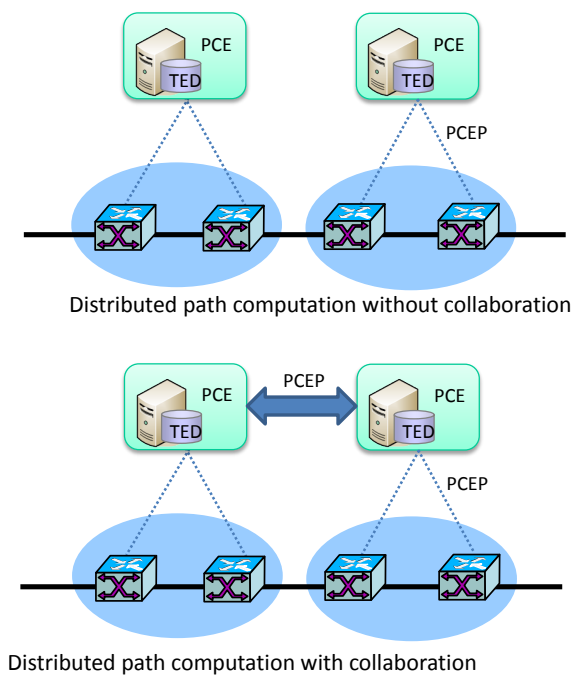

(b) PCE-based Distributed Path Computation

Fig. 3: PCE-based path computation models

els, namely, centralized and distributed. In the centralized model (Fig.3.a), there is a single and centralized PCE that processes all path computation requests from all PCCs. Since this model has a single point of failure, a backup PCE can take over the computation responsibility in case of failure of the working PCE. In the distributed path computation model(Fig. 3.b), several PCEs can be deployed, each one serving requests from a subset of GMPLS controllers, and where computation of paths is shared among the PCEs. Thus, PCEs (handling different switching layers or domains) can compute suboptimal paths with no collaboration (by expanding loose hops at PCE of transit domains), or collaboratively compute endto-end optimum multi-layer and multi-domain paths. To this end, each PCE is responsible for the path computation within its domain or layer.

PCE protocol (PCEP) is used for communicating both PCC and PCE, as well as between PCEs. It is a standard, flexible and extensible interface and protocol, defined in [8]. 


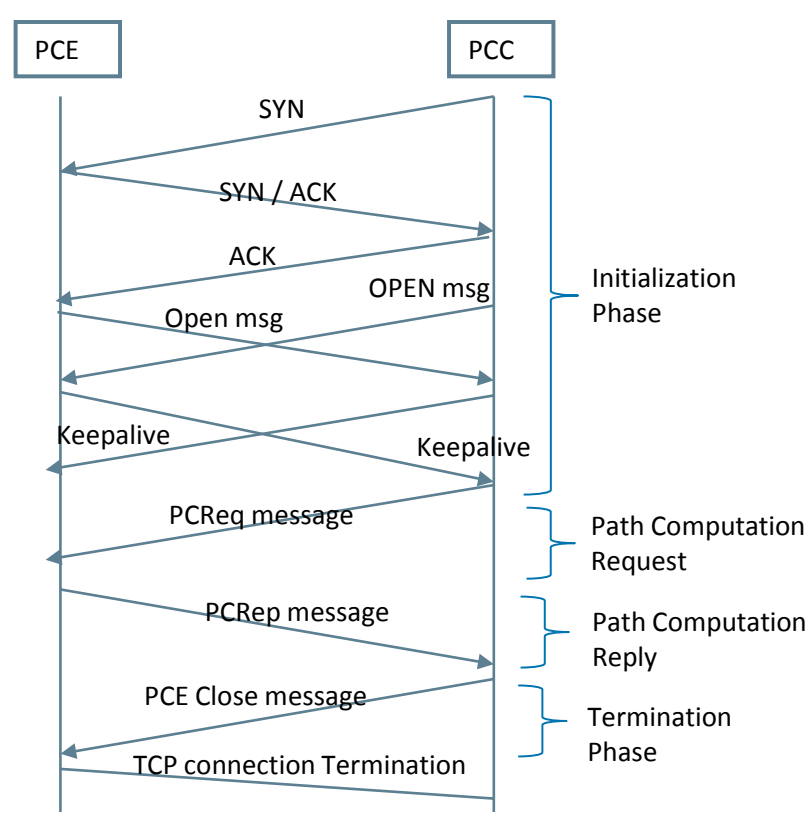

Fig. 4: PCEP session

As shown in Fig. 4, the initialization phase is composed of the 3 way TCP establishment handshake followed by the establishment of the PCEP session through the exchange of OPEN messages, in which keepalive and dead timers, supported objective functions (i.e., path computation algorithms), and capabilities are negotiated. After the initial handshake, a PCC can request point-to-point or point-to-multipoint path computations, by sending a path computation request to the PCE (PCReq message) with a variety of objects that specify the set of constraints and attributes. The PCReq message specifies the endpoints (source and destination node addresses) and objective functions (requested algorithm / optimization criteria), and the associated constraints such as traffic parameters (e.g. requested bandwidth), the switching capability, and the encoding type. It is also possible to include or exclude network nodes, links or whole domains (Include Route Object - IRO - and Exclude Route Object - XRO - respectively), or re-optimize existing paths avoiding resource double-booking using the Reported Route Object (RRO). A PCReq message can request the computation of a path or a set of paths. The use of the Synchronization VECtor (SVEC) list in the PCReq message allows to request synchronized computation, that is, the PCE avoids assigning the same resources to the previous computed paths of the same set. In both cases, each path computation request included in the PCReq requests a specific objective function and defines some constraints. A Global objective function and global list of constraints applied to the complete set of requested path computation can also be performed through Global Concurrent Optimization (GCO), as defined in [9]. Additionally, specific PCEP protocol extensions for RWA and RSA have been proposed in [10], [11] and [12]. If the path computation succeeds, the PCE replies (PCRep message) to the PCC with the computed path/s specified by means of Explicit Route Objects (EROs). Finally, the PCC can close the PCEP and TCP session after the request is served (transient session) or leave it open (permanent session),

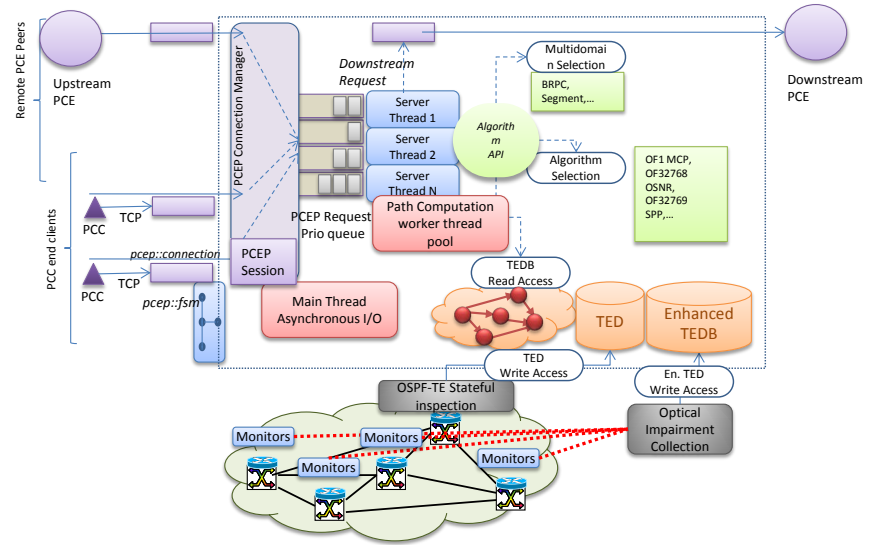

Fig. 5: PCE design with PCE peers developed in the CTTC ADRENALINE testbed

monitored by means of keep-alive messages. Typically, end clients open and close sessions for requests, while sessions between collaborating PCEs are persistent.

One of the key aspects of the Path Computation Element is the synchronization mechanism by which the PCE obtains a copy of the TED in order to perform path computation. Information on network topology and resource status to build the TED may be provided by participating in IGP distribution of TE information, or through an out-of-band synchronization mechanism. In the former, a PCE may collect TE information by maintaining a routing adjacency with a GMPLS controller in the domain (act as a IGP passive listener). In the latter, some mechanism (e.g. a topology server) is used by the PCE to retrieve the TED. Such a mechanism could be either incremental (like IGP) or involving a bulk transfer of the complete TED. In general, out-of-band mechanisms may lead to TED synchronization problems. It is worth noting that the TED may also be enhanced to include additional information obtained from other means.

\section{LIMITATIONS OF GMPLS-CONTROLLED OPTICAL NETWORKS AND PCE-BASED SOLUTIONS}

\section{A. Impairment-aware path computation problem}

The degradation of the optical signal is due to the accumulation of physical impairments whilst the signal travels from the source towards the destination. Such impairments include fiber attenuation, losses, cross-talk, amplified spontaneous emission (ASE) noise, chromatic dispersion (CD), polarization mode dispersion (PMD) and non-linear effects, which may render the quality of the optical signal unacceptable at the receiver. Thus, not all the lightpaths between any pair of source-destination nodes in the network are feasible, that is, have the required level of Quality of Transmission (QoT). To this end, optical layer physical impairments may need to be taken into account by the path computation, in addition to the network topology and resources, in order to provide a path with adequate QoT, which renders the problem even more complex.

An impairment-aware distributed control plane can be classified as distributed routing or distributed signaling [13]. The distributed routing approach requires extensions to the 
standard GMPLS OSPF-TE routing protocol to disseminate information on physical impairments, besides existing TE attributes. For example, [14] proposed translucent-oriented GMPLS protocol extensions to OSPF-TE. Specifically, this work introduced new link and node TLVs (Type/Length/Value) to advertise link and node Optical Signal to Noise Ratio (OSNR). The OSNR is used to capture the impact of the Amplifier Spontaneous Noise (ASE) noise, since the increased number of amplifiers makes the ASE a significant impairment factor to the signal quality. As described in [15], there are two components contributing to the OSNR level estimation: the Link_OSNR that considers the total ASE noise induced by all the (pre- and line) amplifier spans of a link and the Node_OSNR which considers the ASE noise caused by the node booster amplifier.

Therefore, each node maintains a TED with a full picture of the selected physical impairment parameters. Hence, the path computation can provide, as output, the spatial path (nodes and links) that fulfills the adequate QoT. The main limitation of this approach is that the dissemination of optical impairments may cause a significant control plane overhead problem, requiring to maintain a large amount of data at each controller. In fact, no standard routing protocol extensions have been defined so far. Moreover, some impairment information may not be directly mapped to link/node TE attributes disseminated by the GMPLS routing protocol. On the other hand, the distributed signalling approach requires extensions to the GMPLS RSVPTE to gather information on physical impairments parameters, in order to encompass the required QoT whilst establishing the optical connections [16]. The main limitation of this approach is that the information is not available at the path computation time, which may lead to compute unfeasible paths, and therefore, it only allows validating the QoT of the computed path during the signalling phase.

\section{B. PCE-based solution for impairment-aware path computa- tion}

The PCE allows to overcome the limitations of impairmentaware distributed control plane. Physical impairment information is gathered by dedicated monitors and stored in the Enhanced TED (ETED) at the PCE. Thus, impairment information is neither collected nor flooded by the GMPLS controllers. Then, the PCE can use both the TED and the ETED for the path computation. Fig.5, shows the architecture of the PCE developed in the ADRENALINE testbed using the Enhanced TED [17]. The system consists of a main multithreaded asynchronous process, running as a PCEP/TCP server in order to accept and process path computation requests from the PCCs. The PCE can also act as a PCC client to request path computations to other PCEs in other domains or layers. It is composed of a connection manager, a plug-in manager and a path computation engine. The connection manager listens for PCEP/TCP connections, concurrently servicing many clients, using the well known port 4189, accepting PCEP sessions from both end-host clients and PCE peers, asynchronously. The plug-in manager maintains a set of registered plug-ins, which either extend the functionality of the server, or implement the algorithms. The path computation engine executes a RSA algorithm proposed by the authors in [10], [17], to compute elastic paths. It is based on a distance-adaptive, iterative, two-phase approach, combining dynamic spatial path computation (i.e., strict list of nodes an links) based on the TED, and off-line path characterization assuming Coherent Optical Orthogonal Frequency-Division Multiplexing (COOFDM) transmission stored in the ETED, used for dynamic modulation and spectrum assignment, depending on requested bitrate and the physical distance. The characterization of the static paths uses impairment information on fiber attenuation, $\mathrm{CD}$, dispersion slope, and PMD.

\section{Multi-domain path computation problem}

Multi-domain networks are becoming a key component in core transport architectures, given the fact that an operator's network may include several equipment vendors and segmenting the network into domains (as Interior Gateway Protocol -IGP - areas or Autonomous Systems -AS) is a means to increase the overall scalability and/or for confidentiality reasons. A direct consequence of network segmentation is that the GMPLS controllers have a complete view of the network topology and resources within their domain boundaries, but a limited visibility of the other domains. Network segmentation assumes an augmented model in which the exchange of TE information between domains is limited to the dissemination of reachability. Consequently, it is forced to operate on simplified or aggregated topologies. Thus, a source node is not able to compute an end-to-end multi-domain path with an strict list of nodes and links, and only a distributed per-domain path computation can be used. In this mechanism, the source node determines the next domain and the ingress within that domain. Then, it computes the corresponding path segment to the domain boundary, obtaining a strict ERO within its own domain and appending to it (a list of) loose hops for the neighbor domain toward the destination. Next, the path computation moves to the ingress node's controller of the next domain and so forth until the destination domain. During the signaling phase, the node's controllers at each boundary domain expands the ERO. The main limitation of the distributed per-domain path computation is that the egress domain nodes (or Area Border Routers - ABR) determined by the source node may be not optimal, due to the loss of global topology visibility. Let's consider the example of Fig.6.a to illustrate the problem. Node A receives a multi-domain path request (A-Q). Based on the TED of domain 1, Node A computes an ERO composed of node A,C and D (all strict hops, since they are within the same domain) and Q (as loose hop), since it is the optimal path within Domain A. Once the Path message reaches node Q, it expands the ERO, including nodes g,h,i and Q as strict hops, since it is the only available path to reach Q. Indeed, the election of node $\mathrm{Q}$ as egress node of domain A is not optimal, since it has forced selection of a six-hop path.

\section{PCE-based solutions for multi-domain path computation}

Most initial research efforts on the PCE-based multi-domain path computation were targeted to improve or extend the backwards recursive path computation (BRPC) procedure [18]. 


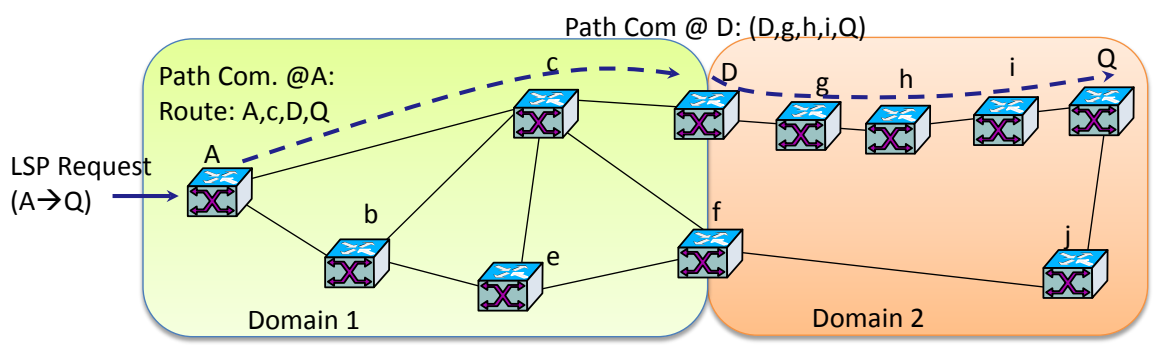

(a) Example of the limitations of per-domain path computation

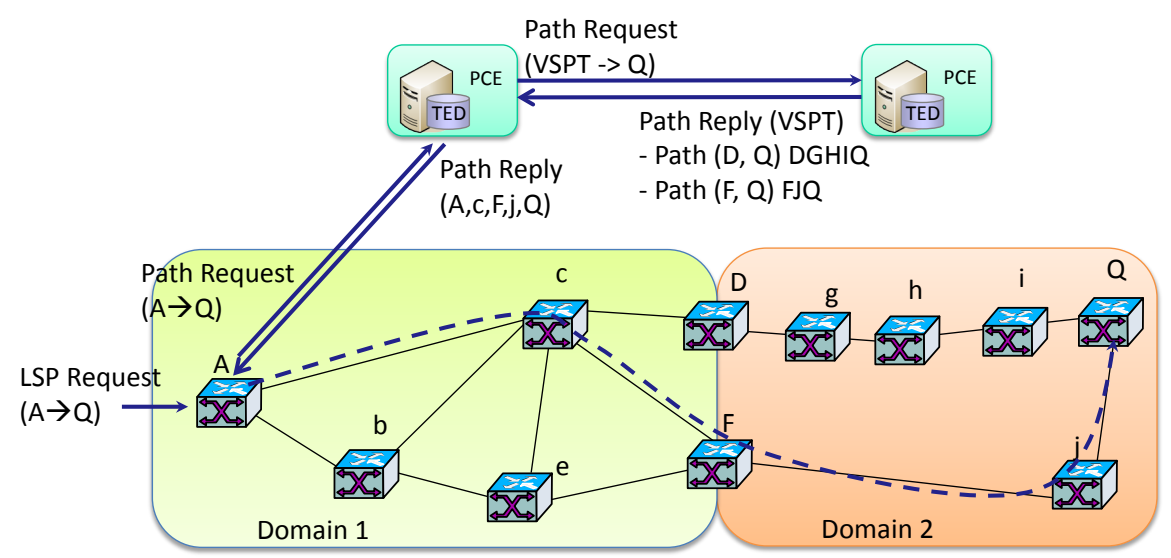

(b) Example of BRPC for PCE-based multi-domain path computation

Fig. 6: Multi-domain path computation

It is based on the discovery and trimming of shortest path trees from domain exit nodes to the destination, using forward and backward requests and responses from the ingress domain to the egress domain. BRPC was standardized as an improvement with regards to the per-domain method. This method assumes that a domain chain (succession of transit TE domains from source to destination) is known in advance. The method relies on dedicated PCEs, which collaboratively compute a multidomain optimum path along the given domain chain. Each PCE is responsible for the path computation within its domain.

BRPC computes the multi-domain path in a reverse way, starting with the destination domain (i.e., in a backward direction). The destination domain PCE computes a virtual shortest path tree (VSPT) from the domain ingress nodes (inABRs in our context) to the destination node. The destination domain PCE sends the computed VSPT to the upstream PCE. This PCE uses this information to compute its own VSPT: computing first a tree from its domain ingress ABRs that are adjacent to the upstream domain (in-ABRs) to each of its domain egress ABRs adjacent to the downstream domain (out-ABRs). Then it selects the optimal path from each of the in-ABRs to the destination node (through any of the outABRs), pruning the suboptimal paths from the VSPT before sending it to its own upstream domain PCE. In other words, the PCE computes its own VSPT from the in-ABRs using the received paths in the VSPT as extended TE links. The upstream domains (i.e., recursive) apply this procedure up to the source domain. Standard BRPC procedure does not consider wavelength continuity constraint in WSON. PCEP extensions for BRPC approaches for WSON were firstly proposed and experimentally evaluated in [11].

For illustration, we consider the example in Fig.6.b. First, node A requests a path computation (A-Q) to the PCE located in its domain. Since node Q is out of the domain, the PCE in domain 1 realizes that the path computation is multi-domain and requests to the downstream PCE a VSPT to node Q. The PCE in domain 2 computes an optimal path from the two inABRs to destination node, namely (D-g-h-I-Q) and (F-j-Q) The resulting VSPT is sent to the PCE in domain 1, which computes first the set of paths between the source node (A) and the two out-ABRs (D,F), obtaining (A,c,D) and (A,c,F), and then uses the received information to compute the optimal path from the source to destination, selecting (A-c-F-j-Q).

It is worth noting that it could also be feasible to build the VSPTs in the forward direction (i.e., from the source domain PCE to the destination domain PCE), as proposed in [19]. In this case, the source domain PCE may compute an inverseVSPT (i-VSPT) from the source node to the domain egress nodes. Then, the source domain PCE may send the computed i-VSPT to the downstream PCE to compute its own i-VSPT (the shortest paths from the source node to all domain egress nodes). The downstream domains would recursively apply this procedure up to the destination domain. Finally, the destination domain PCE would compute the optimum path from the received i-VSPT to the destination node. This approach would require that the destination domain PCE informs to the source domain PCE (i.e., in the forward direction) about the computed path. Since the PCE protocol (PCEP) is mainly oriented to the exchange of PCReq/PCRep messages, PCEP would require complex protocol extensions to allow an upstream PCE to 


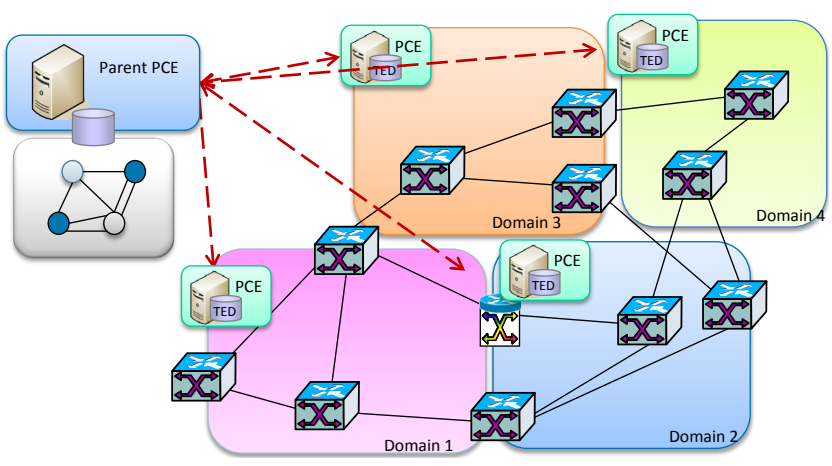

Fig. 7: Example of Hierarchical PCE (H-PCE)

inform about the computed i-VSPT to a downstream PCE. For this reason, this approach has not been considered for standardization.

BRPC attains optimal path computation if the sequence of domains is known. This method could also be applied to meshed domains but it becomes very complex. Lately, an approach for arbitrary domain meshes named hierarchical PCE (H-PCE) is being proposed [20], in which the domain selection is delegated to a higher-level entity (a parent PCE). In this approach, a two-level H-PCE is considered. As shown in Figure 7, a child PCE (c-pce) is deployed at each domain, which, for example, may correspond to an AS with a single OSPF-TE area. A single parent PCE (p-PCE) is deployed, being responsible for the domain sequence selection using aggregated topologies of the domains. Each c-PCE establishes a persistent PCEP protocol adjacency with the p-PCE, and the adjacency is used for in-band notifications, topology aggregation updates, and segment expansions. It is the responsibility of each c-PCE to perform path computations (e.g., upon request from the p-PCE) within its domain, using the regular TED and algorithms. When these computations are part of an end-to-end path computation they are referred to as segment expansions.

Parent PCE builds a simplified topology of the domains through two main topology aggregation mechanisms:

- Virtual node aggregation: Each domain is presented as a virtual node. In the example of Fig.8.a, each domain is seen as a node for the parent PCE. Then, the ABRs of each domain are seen as ports of the virtual nodes, and are connected through inter-domain links. The main drawback of this mechanism is that it hides internal connectivity issues, leading to sub-optimal domain sequence selection.

- Virtual link aggregation: Network domain's internal connectivity can be dynamically mapped to a mesh of virtual links. In the example of Fig. 8.b, the child PCE in domain 1 computes paths between border nodes that appear as virtual links in the parent PCE [21]. The main drawback of this approach is that it requires large amount of information and frequent updates.

As shown in Fig. 9, the path computation procedure is initiated by an PCC end client which requests a multi-domain path computation to the PCE in its domain, who forwards the request to the parent. The parent identifies the source and destination domains and requests a Virtual Shortest Path Tree (VSPT) to the PCE within the destination domain and an
inverse-VSPT (i-VSPT) to the PCE within the source domain, with the paths from the destination domain entry border nodes to the destination endpoint (VSPT), and the tree containing the paths from the source end point to the source domain exit border nodes (i-VSPT). The parent extends the aggregated topology with virtual links induced from the paths of the VSPT/i-VSPT, and performs a core path computation, based on the directed graph formed by the virtual edges. For each core ERO segment, a request is sent to the corresponding child to expand the segment. Finally, the parent combines the domain segments for an end to end path and sends the reply to originating child PCE / PCC.

Aggregation and topology summarization procedures by which a domain is aggregated are complex, and represent a trade-off between the amount of information and optimality, in terms of domain internal connectivity and available resources but also in terms of capabilities and aggregation opportunities. For example, multi-rate systems require new aggregation and summarization methods, since heterogeneous wavelength data rates prevent unifying bandwidth management, and the computation of TE link attributes such as unreserved bandwidth or LSP bandwidth in a straightforward manner. Moreover, specific aspects of the optical technology, such as node capabilities, different supported modulation format and data rates, transceiver or regenerator availability etc. as well as the need to take into account physical impairments to guarantee the quality of transmission further increase the complexity and the volume of topological (TE attributes) information.

\section{E. Multi-layer path computation problem}

Multi-layer transport network integrating both packet and optical circuit switching technologies (e.g., packet switching capable - PSC for MPLS-TP, and lambda switching capable - LSC for WSON), leverages the high-bandwidth transport capacity and deterministic performance provided by the optical circuit switched technology, as well as the efficient traffic aggregation (grooming) and statistical multiplexing provided by packet switched networks. Particularly, the purpose of the traffic grooming is to merge/group low-speed and flexible higher-layer LSPs (e.g., PSC LSPs) with small bandwidth requirements into new or already established high-speed lowerlayer connections with coarse bandwidth (i.e., LSC tunnels).

To fully exploit the advantages provided by the traffic grooming it is necessary to promote the cooperation among the layers by means of a peer control plane interworking model. To do this, a GMPLS unified control plane is adopted, where a single control plane instance (routing and signaling) is applied in a ubiquitous way to control all the switching layers within the same domain. Thus, each node has the global view of all the layers including the network topology and resource availability in the TED repository. The TED information is then used as input by advanced multi-layer path computation algorithms to dynamically provision LSPs across all the layers favoring the grooming decisions [22].

In a multi-layer network, a LSP must initiate and terminate at the same specific layer and may traverse one or more lower layers. When a LSP is set up within a layer from a layer border 


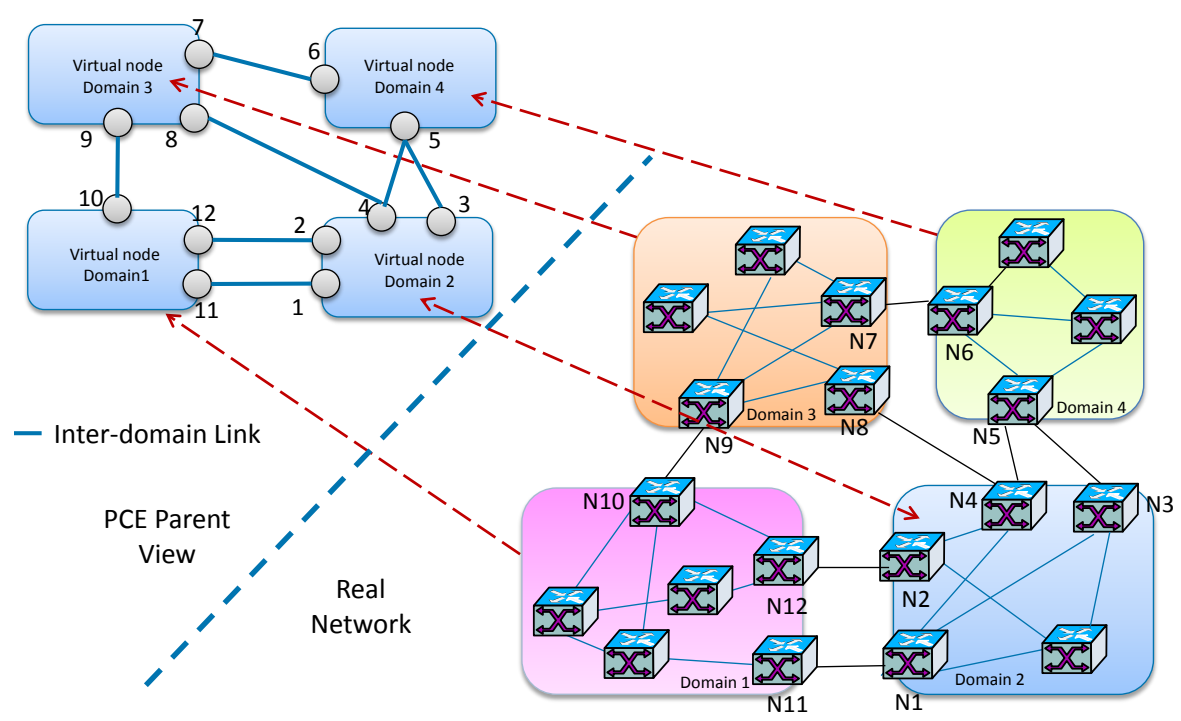

(a) Example of virtual node aggregation

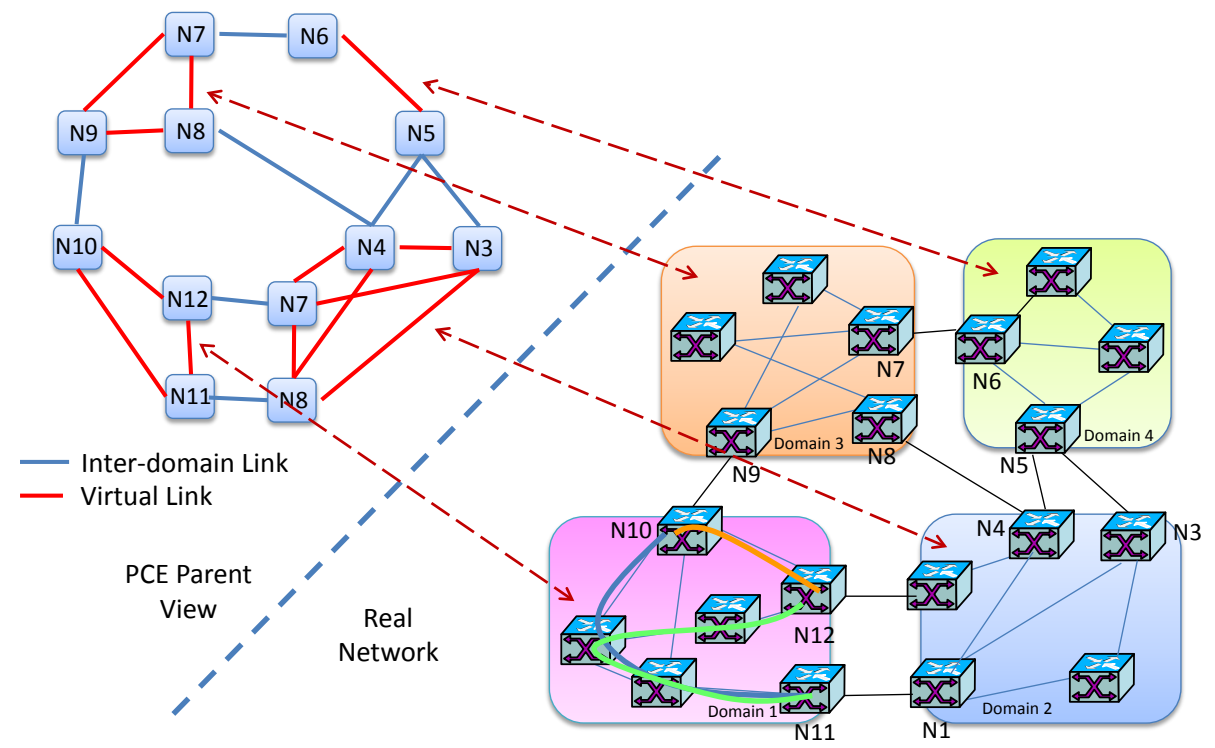

(b) Example of virtual link aggregation

Fig. 8: Network domain aggregation mechanisms in H-PCE

node to another, the established LSP can be then used as a data link for the upper layer [23]. In the GMPLS context, this link is referred to as a Forwarding Adjacency (FA) TE link, and the corresponding LSP is the FA LSP. The attributes of a dynamically created FA TE link (e.g., bandwidth, TE metric, etc.) are inherited from the associated FA LSP, and flooded by the same OSPF-TE protocol instance. The creation of FA TE links facilitates other nodes to use them when computing paths performing the grooming decisions since multiple higher layer (e.g., PSC) LSPs may be grouped over a single lower layer (e.g., LSC) FA tunnel. This signalling mechanism is known as hierarchical signaling.

Let's consider the example of Fig.10 to illustrate the computation and provisioning of a multi-layer path with a GMPLS unified control plane with PCE. The objective is to request a PSC LSP between $\mathrm{H} 1$ and $\mathrm{H} 2$. First, in order to dynamically provision the requested PSC LSP, the source GMPLS controller $(\mathrm{H} 1)$ requests to the PCE the computation of a multi-layer path between $\mathrm{H} 1$ and $\mathrm{H} 2$. Upon reception of the path computation response with the ERO (H1-L1-L2$\mathrm{H} 2$ ), $\mathrm{H} 1$ initiates the provisioning of the LSP. It realizes that it is at the edge of the PSC layer (i.e., TE link H1-L1 has different switching types at the edges: PSC and LSC). Thus, the H1 needs to set up an LSC FA LSP (PATH_1 / RESV_1) to the other layer edge (i.e., H2). The establishment of such a LSC FA LSP induces the creation of the PSC FA TE link between $\mathrm{H} 1$ and $\mathrm{H} 2$. To this end, it is necessary to exchange the unnumbered interface identifiers using the LSP Interface Id (LTII) during the FA LSP establishment. That is, the ingress and egress LSRs exchange the interface identifiers used to unambiguously identify the created FA TE link. Once the LSC FA LSP is established, the attributes (e.g., unreserved 


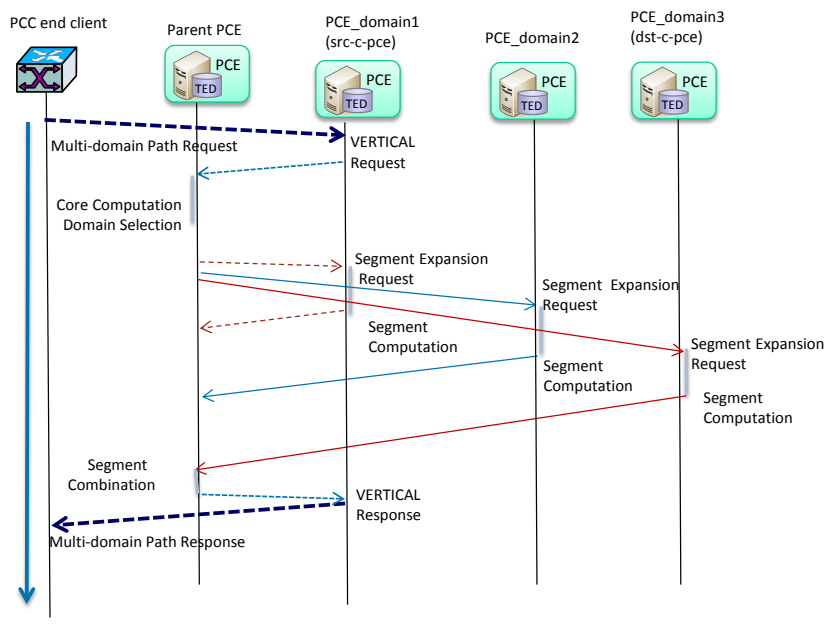

Fig. 9: Example of H-PCE multi-domain path computation

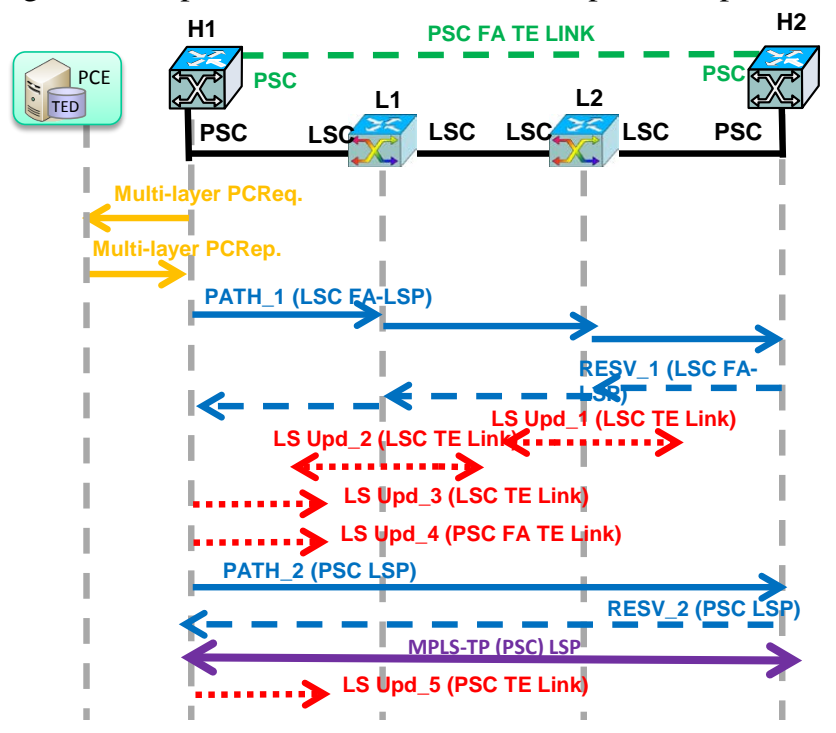

Fig. 10: Example of PCE-based multi-layer path computation and hierarchical signaling with unified control plane

bandwidth) of the traversed optical physical links are updated (i.e., LS Upd_1, Upd_2 and Upd_3 OSPF packets). Next, the created PSC FA TE link is flooded (LS Upd_4) by the source H1. Most of the FA TE link attributes are inherited from the associated LSC FA LSP. For example, the unreserved bandwidth of the PSC FA TE link is set to the data rate supported by the DWDM transceiver which is allocated by the LSC FA LSP. On the other hand, the link cost (TE metric) is administratively set to a value which favors grooming objectives. Finally, the PSC LSP between H1-H2 is established through the PSC FA TE link (PATH_2 / RESV_2), and thus, an OSPF-TE LS Update packet (LS_Upd_5) is flooded to update the unreserved bandwidth of the FA TE link.

\section{F. PCE-based solutions for multi-layer path computation}

In [24], the first implementation and lab trial demonstration of a unified control plane for multi-layer (MPLS-TP/WSON) networks was presented. However, a unified control plane may be difficult to be deployed in multi-layer production networks involving different vendors' equipment. Alternatively, under an overlay interworking control model, each data plane switching layer has its own control plane instance. The interaction model assumes a client-server relationship, with very low trust relationship between the control plane layers. Indeed, this model mandates a strict separation of the respective network control planes (including their addressing spaces), and strictly limits the control exchange to signalling information. In other words, the routing and signalling protocols of each control plane layer act independently. Hence, a packet switching node is not able to compute an end-to-end path traversing the underlying optical layer since it does not have the topology and resource information of such a switching layer. The lack of such multi-layer network topology information prevents to globally optimize network resource utilization. Network resource optimization can only be performed at each layer independently, leading to an increased blocking probability, since the region boundary nodes selected by the source node are not optimal, as happened with the multi-domain path computation. Path optimality (i.e., path computation output in the form of a strict ERO object including each layer subobjects) requires coordinated PCE-based path computation or full topology visibility, by means of one the following approaches:

- Single PCE with topology visibility of all layers (Fig.11.a): Either in a single combined TED or in a joint processing of each layer TED. In this approach, only the PCE knows the full topology, not the routing agents in each layer.

- Per-layer PCE in a collaborative setting (Fig.11.b): Each layer PCE knows its layer topology, but needs to ensure that the optimal region boundary nodes are selected. To this end, it uses PCEP-based procedures conceptually similar to BRPC or H-PCE based solutions in a multidomain context [25] to ensure optimality, as explained in section III-D.

In general, multi-layer TE relies on both an optimal multilayer path computation and the automated provisioning of all involved layers. Inter-layer automated provisioning depends on the ability to provision all (server/client) layers, in one of the following two approaches:

- Hierarchical signaling: The establishment of a client LSP triggers the establishment of a server layer connection at region boundaries, using for example the Generalized UNI [26]. This approach is conceptually similar to the hierarchical signaling mechanism used in the peer model explained in Sec. III-D. It is also necessary to exchange the unnumbered interface identifiers, as done during the FA LSP establishment.

- Layered provisioning: An entity named Virtual Network Topology Manager (VNTM) is able to coordinate the layered establishment of server and client connections.

Both approaches are based on the promotion of a server connection as a client layer (logical) TE link. Thus, it is similar to the Forwarding Adjacency concept considered in a unified control plane. The dissemination of this logical/virtual TE link is driven by the server layer connection head-end LSR or by the VNTM. To better illustrate the use of the VNTM for multi-layer path computation with layered provisioning, 


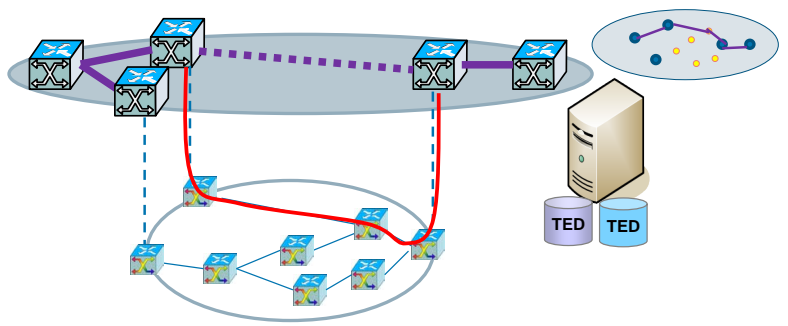

(a) Single PCE with topology visibility of layers

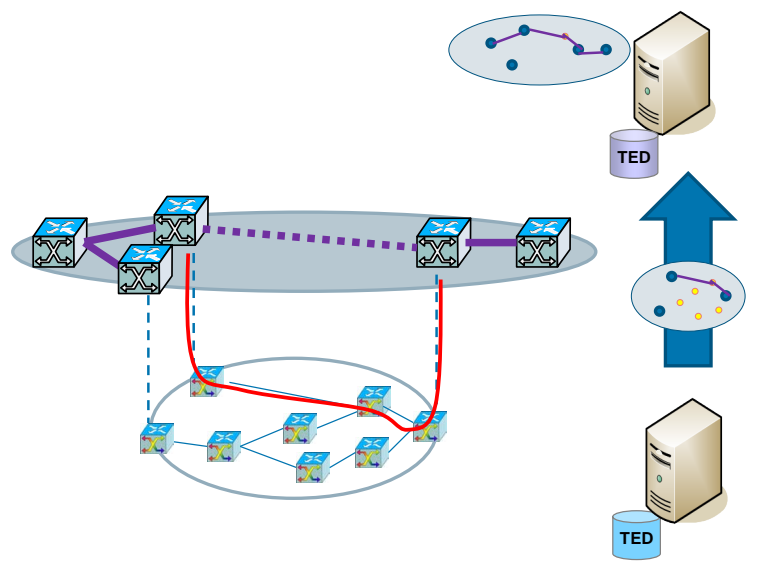

(b) Per-layer PCE in a collaborative setting

Fig. 11: PCE-based multi-layer path computation in an overlay model

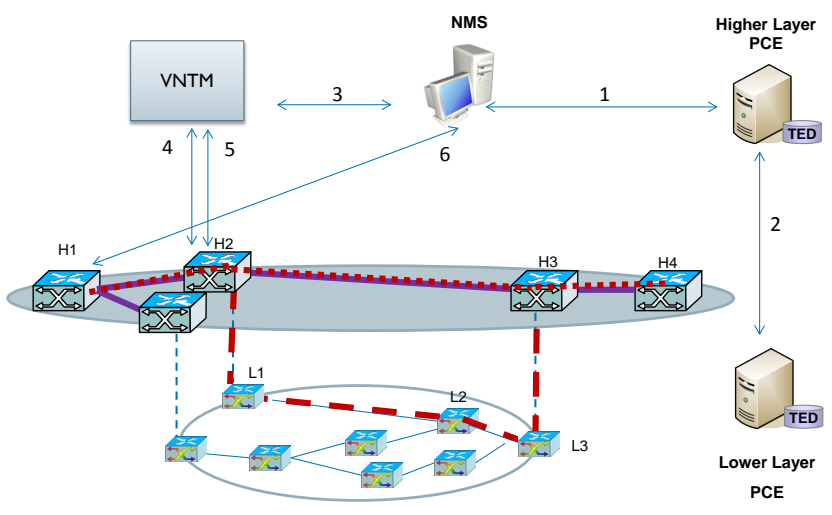

Fig. 12: Example of PCE-based multi-layer path computation with layered provisioning

let us consider the example of Fig.12. First, the NMS requests the computation of a multi-layer path between $\mathrm{H} 1$ and $\mathrm{H} 4$ to the PCE located in the higher layer (e.g., packet). The higher layer PCE collaboratively computes and end-to-end optimum multi-layer path with the lower layer PCE, using, for example, BRPC. After the path computation, the higher layer PCE sends to the NMS the computed multi-layer path (H1-H2-L1-L2-L3$\mathrm{H} 3-\mathrm{H} 4)$. The NMS realizes that the path includes a lower layer (optical) path (H2-L1-L2-L3-H3). Thus, the NMS requests to the VNTM the provisioning of the optical connection and its promotion as virtual packet TE link. The VNTM is responsible for requesting to $\mathrm{H} 2$ (head-end of the optical connection), first, the provisioning of the optical path (provisioned through the exchange of RSVP-TE Path and Resv messages), and upon the reception of the successful reply, the dissemination of the optical connection as a new virtual packet TE link between $\mathrm{H} 2$ and $\mathrm{H} 3$ (disseminated through the exchange of OSPFTE TE LSA messages). Finally, the VNTM notifies the NMS about the successful creation of the virtual TE link, and the NMS proceeds to request the provisioning of the higher layer (packet) path to node H1 (head-end of the packet connection). Once the packet connection is established, the node $\mathrm{H} 1$ notifies to the NMS.

\section{Limitations OF THE PCE}

\section{A. TED synchronization}

As described in Sec.II, a PCE operates with network state information (topology and resources) collected in the TED provided by a synchronization mechanism. Initial synchronization mechanisms were based on IGP passive listener for intra-domain TED. However, it is also required to discover neighboring domains, border nodes, inter-domain links or peering PCE addresses. Thus, new synchronization mechanisms are being proposed to extend current mechanisms, such as embedded PCEP notifications [27] to obtain the TED by PCEP, or new protocols such as BGP-LS [28] to obtain the TED by BGP peering.

A PCE does not store the state information of the computed paths (i.e., stateless), and a request or set of requests is processed independently of each other. Thus, a (stateless) PCE computes paths based uniquely on TED information which may not be synchronized with the actual network state, leading to an increase in the blocking of the connections during the provisioning. Let us consider the example of Fig.13 to illustrate the problem when the TED is synchronized by OSPF-TE mechanism. This mechanism allows synchronizing all the nodes's TED repositories within a given time after a network state change, referred to as the routing convergence time (in this example we assume whenever there is a change in the network state, a link update state is disseminated just after the change). However, if multiple connections are set up almost simultaneously (e.g, LSR2-LSR3, and LSR1LSR3), it is likely that the PCE computes LSPs that share the same wavelength in a common link (e.g.,Lamba A in link LSR2-3), since the PCE uses the same TED for both path computations (The OSPF-TE link state update from the first provisioned LSP arrives to the PCE after it computes the second LSP). Therefore, the second LSP will be blocked during the provisioning. In general, two path computation requests must be separated, at least, more than the OSPFTE routing convergence time to ensure that the PCE operates with a fully updated TED. This requirement could be satisfied for dynamic provisioning, since it is unlikely to work under 


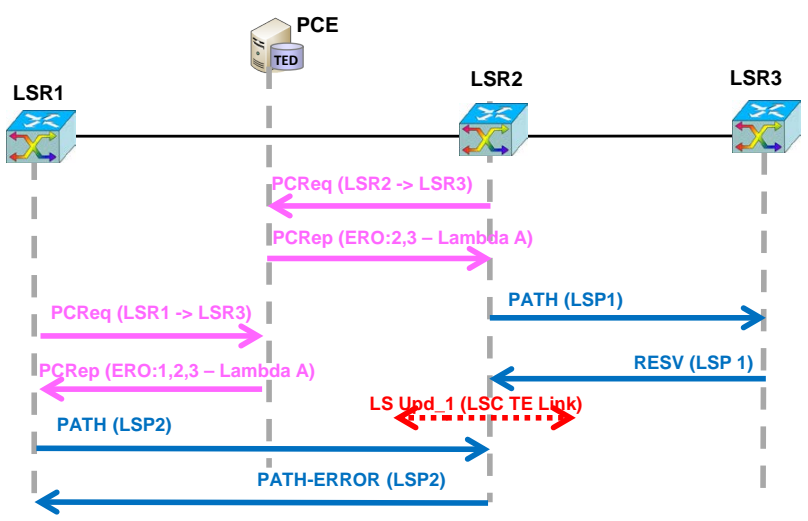

Fig. 13: Example of impact of outdated TED on PCE-based path computation

highly dynamic traffic scenarios. However, this requirement cannot be satisfied for dynamic restoration, where the failed LSPs are dynamically restored by backup paths computed by the PCE. Specifically, the ingress node of each disrupted LSP sends a backup path request to the PCE. This may result on simultaneous path computation requests if multiple LSPs were interrupted, that may reduce the restorability.

The accuracy of the TED may also depend on how often link state updates are sent. In general, two flooding policies can be applied, aiming at minimizing the routing protocol overhead, classified into either time-triggered or event-triggered schemes. In the time-triggered schemes, the link state updates are generated periodically, based on a specified interval of time between two successive link state updates originated at a given node. As for the event-triggered schemes, a new link state update is generated based on a specified unreserved bandwidth variation (i.e., a threshold) on a given link. It is worth noting that the use of either time-triggered or event-triggered flooding policies highly affects the accuracy of the node's TED information. Therefore, a tradeoff between the generated volume of control information and the network performance is clearly stated.

Two solutions have been proposed to minimize the impact of outdated information on the TED:

- The PCE may store (e.g., in the enhanced TED) for a limited period of time some information from recently computed paths so that it avoids the use of the same resources for other LSPs, as shown in Fig.14.a. That is, a limited form of statefulness is applied, known as contextawareness [6], [29]. In [30], Proactive PCE is proposed, in which the PCE itself updates immediately the TED after the path computation, assuming that the computed LSP is already established and possible errors occurring during the signalling phase are detected and notified to the PCE by the source node.

- The PCE may retain for a limited period of time some LSP request, and process concurrently all the received requests when the timer expires [31], as shown in Fig.14.b.

\section{B. Lack of global LSP state}

The lack of global LSP state information (e.g., LSP route and reserved resources) may result in sub-optimal PCE algorithms. For example, the non-linear effects of optical fibers such as Cross-talk (XT), Cross-Phase Modulation (XPM) or Four-wave mixing (FWM) generate adjacent wavelength/ frequency slot interference that may cause the provisioning of a new optical connection to degrade the quality of service (QoS) of the in-service connections. Thus, impairment-aware RMSA algorithms should compute new paths that ensure an acceptable QoS of the existing ones. To this end, impairmentaware RWA or RSA algorithms must also know the existing LSPs in order to recompute the considered QoS parameters (e.g., Q factor) of LSPs adjacent to the computed path, and verify whether it still satisfies a given threshold.

Another example is the minimal perturbation problem. The objective of this algorithm is to route a demand along the path that requires the lowest number of preemptions (in case no bandwidth is available). Without knowledge of LSPs, preempting low-priority LSPs based on the minimum number of links may not result in the smallest number of LSPs being disrupted, as can be appreciated in the example of Fig.15. In this example, the PCE receives a new path computation request (LSR1,LSR7). Since, there is no path with enough available bandwidth, the algorithm must preempt some LSPs to free some bandwidth. If the PCE computes the path without global LSP state, the PCE will select the path (LSR1-LSR2-LSR3LSR7), since it minimizes the number of links. However, it is not the optimal path, since this would require to preempt three LSP (LSP1, LSP2 and LSP3). If the PCE would have computed the requested path with global LSP state, it would have selected the path (LSR1,LSR4,LSR5,LSR6,LSR7), that despite having one additional link, it only requires to preempt one LSP.

\section{Control of path reservations}

The PCE lack of control of path reservations (e.g modification or rerouting) may also result in sub-optimal PCE algorithms. Let us consider the example of Fig.16. The PCE receives a path request for LSP1 (LSR1,LSR6) requiring $5 \mathrm{~Gb} / \mathrm{s}$, and it computes the shortest path (LSR1-LSR3-LSR5LSR6) with available bandwidth. Later, the PCE receives a path request for LSP2 (LSR2,LSR7) requiring $7 \mathrm{~Gb} / \mathrm{s}$. As can be appreciated, there is no path with enough available resources. Therefore, the PCE fails to provide a path for LSP2 and the connection is blocked. This problem could be solved if the PCE could optimize some of the existing LSPs during the path computation process. Thus, LSP2 would not fail if the PCE could re-route LSP1 through LSR1-LSR4-LSR5-LSR6. Then, the PCE could compute a path for LSP2 (LSR1-LSR3LSR5-LSR6).

\section{INTROdUCtion to the STATEFUl PCE}

A stateful PCE allows for efficient path computation and increased path computation success, considering both the network state (TED) and the LSP state (LSPDB) (i.e., set of computed paths and reserved resources in use in the network), as shown in Fig.17. Thus, a strict synchronization mechanism is required to allow the stateful PCE to build the global LSPDB, based on the local LSPDBs stored in the GMPLS controllers. In [32], it is proposed to extend PCEP with a new 


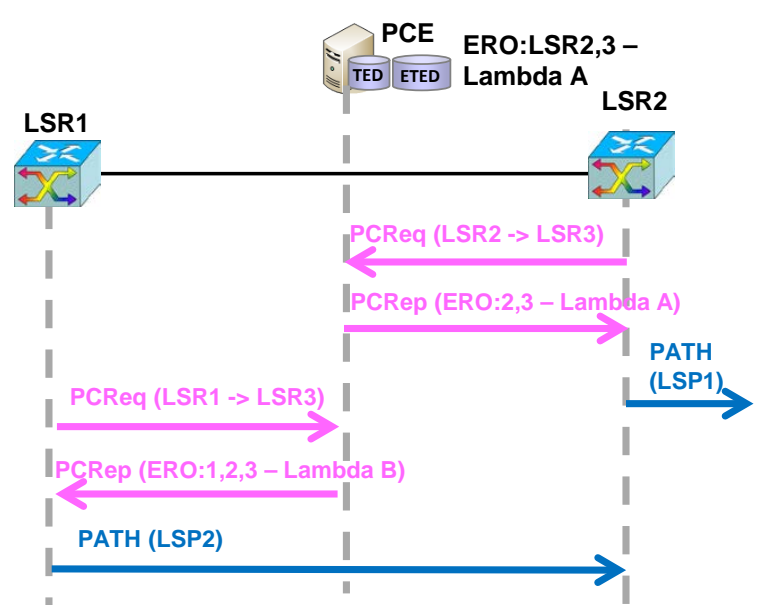

(a) Context-awareness

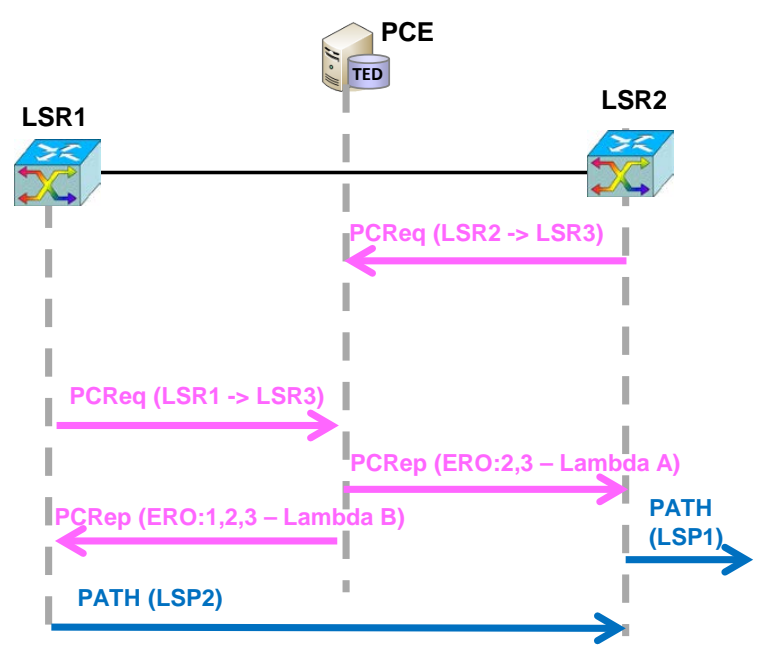

(b) Concurrent path computation

Fig. 14: Solutions to minimize the outdated TED

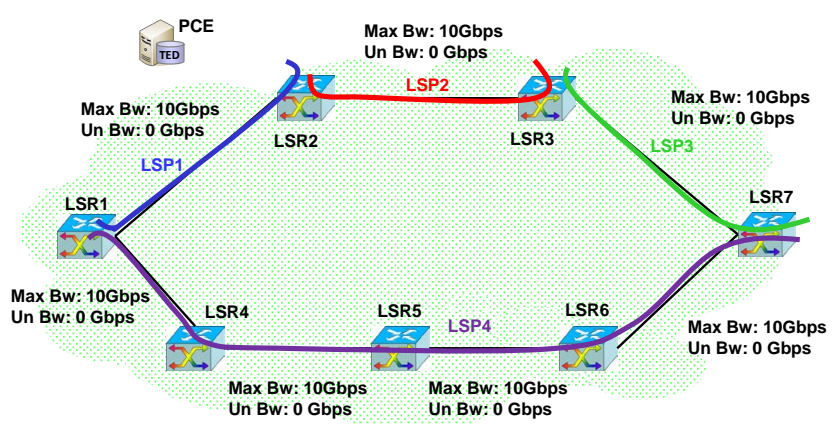

Fig. 15: Example of impact of lack of global LSP state on PCE-based path computation

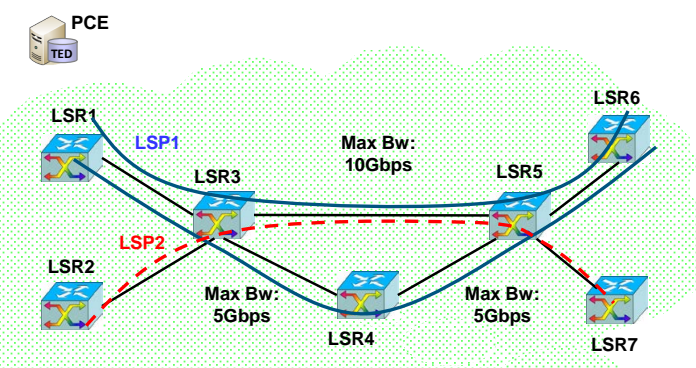

Fig. 16: Example of impact of lack of control of path reservations on PCE-based path computation

message named Path Computation State Report (PCRpt) to allow a stateful PCE to learn the LSP state (i.e., the PCCs report about the state of the LSP whenever there is a change in their LSPDB, or under request). This PCE is known as passive stateful PCE.

A passive stateful PCE maintains a global LSPDB that is only used as input for new path computations. Thus, a passive stateful PCE does not have control (modification, rerouting) of path reservations stored in the LSPDB. To this end, an active stateful PCE allows for optimal path computation considering the LSPDB, but not only as an input of the path computation

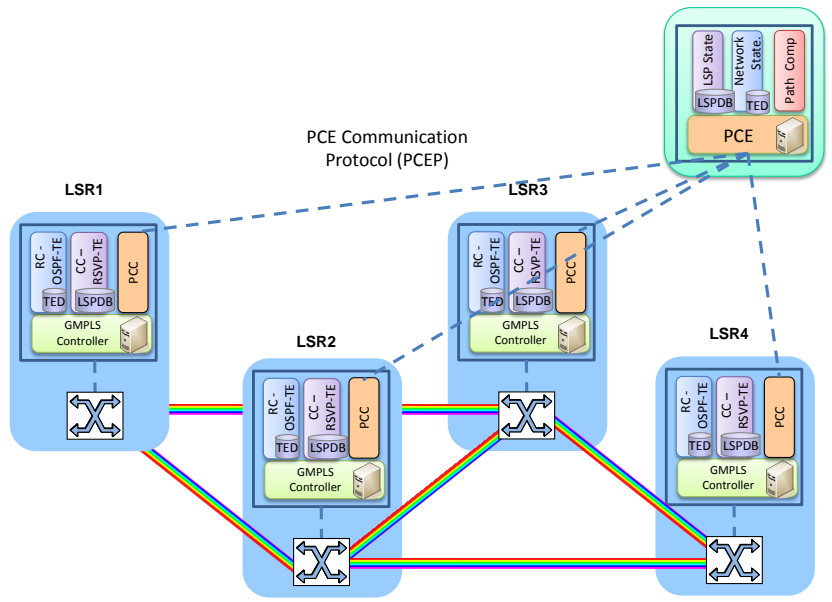

Fig. 17: Example of GMPLS-controlled optical network with stateful PCE

process, but also for the control of the state (e.g. rerouting) of the stored LSPs. Since under distributed control LSPs are only managed by the GMPLS controllers, this approach requires that the GMPLS controllers temporarily delegate the control of a set of active LSPs to an active stateful PCE. A delegation mechanism based on the PCRpt message is proposed in [32] for PCEP. Moreover, an active stateful PCE can also request the modification/rerouting of the existing LSPs (e.g., to reoptimize the path) to the source GMPLS controllers (headend PCC of the connection). To this end, [32] also proposes a new PCEP message named Path Computation Update Request (PCUpd) sent from the PCE to the PCC. Upon reception of a PCUpd message, the PCC triggers the modification of the LSP in the GMPLS controller through the exchange of the Path and Resv messages. Fig.18.a provides an example of a LSP initiated by the PCC, but later modified (e.g., increase of the allocated frequency slot in a SSON) by the PCE.

Additionally, an active stateful PCE could not only modify existing LSPs but also setup/release new LSPs [33]. It is known as active stateful PCE with instantiation capabilities. In 
[34], it is proposed to extend PCEP with a new message named LSP Create Request (PCCreate) to allow PCE to request the creation of new LSPs to the GMPLS controllers. Deletion of PCE-initiated LSPs is performed through a particular case of the PCUpd message. Fig.18.b provides an example of a LSP created and later removed by the PCE.

\section{PCE DEPLOyMENT MOdels IN SOFTWARE Defined NETWORKS}

Software-defined networks (SDN) are based on three key pillars, as shown in Fig.19. First, the control plane is decoupled from data plane and logically centralized in a single controller. This controller maintains a detailed, global, updated and unique view of the network state (TED) and connection state (LSPDB). This single controller is responsible for computing paths in a centralized way and for provisioning the connections in a centralized vertical manner (i.e., without requiring horizontal coordination among the nodes). Second, a standardized interface between the controller and the data plane, for example based on the OpenFlow protocol [35] for connection provisioning and network discovery. In brief, OpenFlow allows to identify and abstract the common set of functions from switches (both packet and circuit) and routers, and offer them in an standardized way to the centralized controller [36]. Consequently, OpenFlow provides an open protocol to program and query flow tables and cross-connects. Third, a well-defined API in the centralized controller to allow applications to control the network. Currently, standard OpenFlow (v1.3) does not support optical circuit switching features (neither for WSON nor SSON). OpenFlow protocol extensions were presented and experimentally evaluated in [37], [38] for WSON, and in [39], [40] for SSON, extending the circuit addendum 0.3 for OpenFlow v1.0 [41].

On the other hand, this formal decoupling of the path computation in well-defined PCEs with an open and well-defined PCEP, facilitates the deployment of PCEs within diverse control plane architectures (beyond the original MPLS/GMPLS), in which it is then possible to offload computations to dedicated PCEs and benefit from distributed computations amongst collaborative PCEs. In view of this, the particular integration of the PCEs within the SDN/OpenFlow centralized control model becomes an opportunity, since it would allow to reuse the know-how of algorithms developed in the scope of PCE in the OpenFlow controller [42].

A stateless or passive stateful PCE can be integrated with an OpenFlow controller as an application of the controller (Fig.20.a). In this scenario, the PCE can be directly an application on top of the OpenFlow controller. The PCE builds its local TED and the LSPDB through the defined API with the OpenFlow controller. It could also be formally decoupled from the OpenFlow controller using a PCC application on top of the controller that requests path computations to the PCE [43], as shown in Fig.20.b. The TED and LSPDB must be requested back to the OpenFlow controller (e.g., obtained from a dedicated topology server or through the PCC). It is worth nothing that the PCE cannot maintain the TED or the LSPDB by updating immediately the TED or the LSPDB after the path computation.
Finally, if the PCE is an active stateful PCE with instantiation capabilities, the PCE becomes a full controller (provisioning, modification and release of LSPs) and therefore, it could be integrated with the SDN/OpenFlow controller (Fig.20.c). In this approach, interfaces are internal and both components have access to a single instance of the TED and LSPDB. Moreover, this full integration is motivated by the fact that having common, shared date structures and state simplifies concurrent access and updates. In [44], it is presented the first work that considered an integrated stateful PCE/OpenFlow controller for flexi-grid networks.

\section{CONCLUSIONS}

We have overviewed the PCE architecture and how it can mitigate some weaknesses of GMPLS-controlled WSON/SSON. Then, we have identified some of its own limitations and the way they are being addressed, along with the advanced deployments in SDN/OpenFlow. This paper has identified two main trends. On the one hand, the PCE is moving from stateless to stateful. The rationale behind this is that it overcomes the limitations of the stateless PCE in GMPLS-controlled optical networks that lead to increase the path computation blocking or to sub-optimal path computations, such as the outdated TED, lack of global LSP state, and lack of control of path reservations. A stateful PCE allows optimal path computation and increased path computation success, considering both the network state and the LSP state. Additionally an active stateful PCE can also setup, reoptimize and release data connections. However, the topic of stateful PCE is very recent, requiring significant research effort, since there are some open issues; first, in large networks the maintenance of a LSP database can be non-trivial and may require substantial control plane resources. Thus, the stateful PCE requires a reliable synchronization mechanism. Second, in networks with several PCEs, the path computation and LSP state information are distributed among PCEs. Thus, the stateful PCE would require synchronization of the LSPDB and coordination of the path computation by communicating with each other. Third, path computations considering both TED and LSP databases would be highly complex, requiring large amounts of computer processing resources.

On the other hand, the second trend identified in this paper is that PCEs are being integrated within other control paradigms outside their original scope (MPLS/GMPLS), such as SDN/OpenFlow. The formal decoupling of the path computation, from the rest of the control plane, in centralized and dedicated PCEs with an open, standard and featured interface and protocol (PCEP), allows more flexibility in the deployment of PCEs within SDN architecture. It is then possible to offload computations to dedicated PCEs and benefit from distributed computations amongst collaborative PCEs. Thus, the particular integration of the PCEs within the SDN/OpenFlow centralized control model becomes an opportunity, since it would allow reuse of the know-how of algorithms developed in the scope of PCE in the OpenFlow controller. 


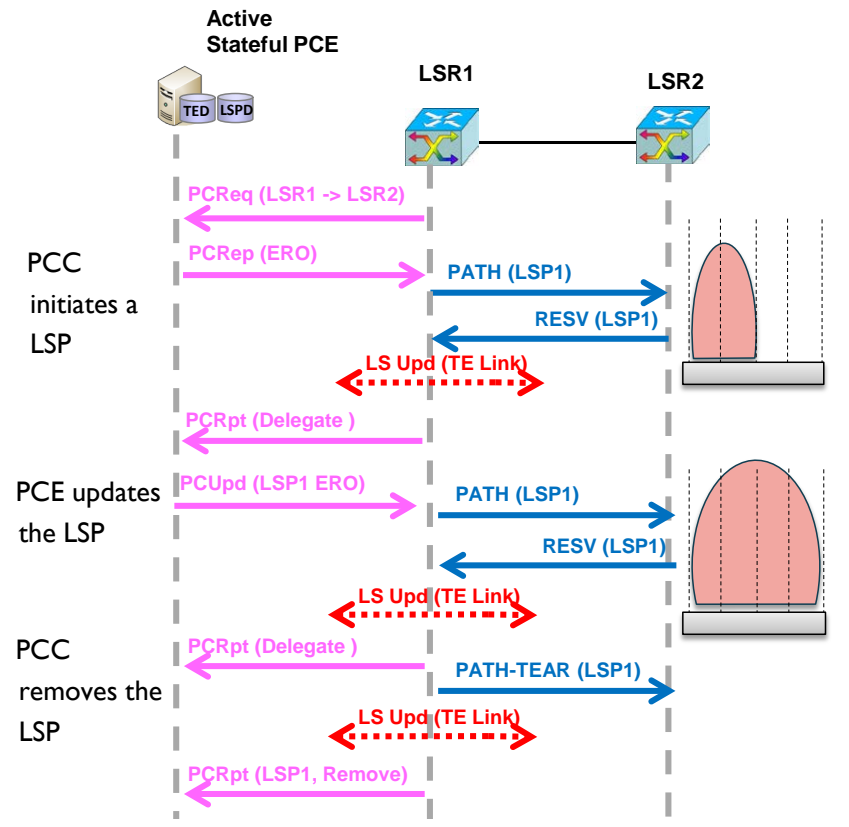

(a) without instantiation capabilities

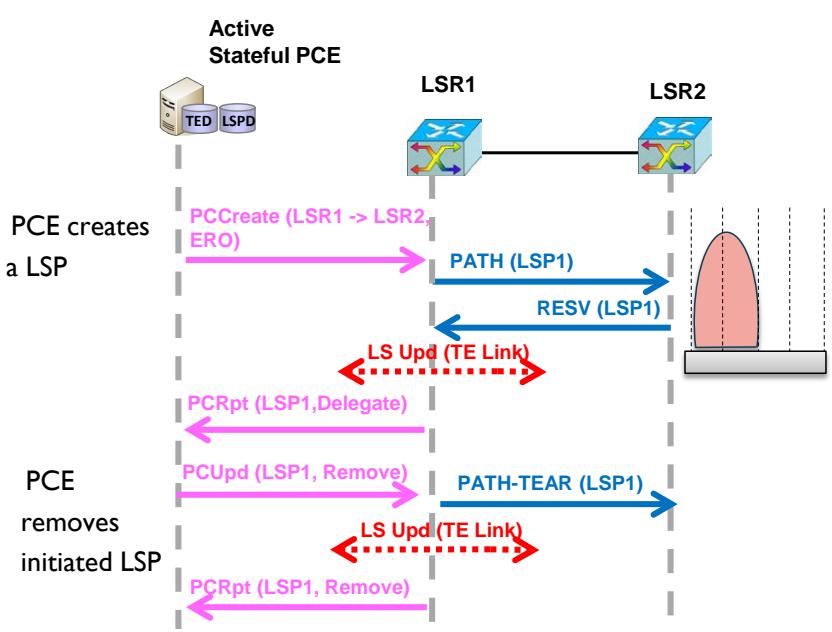

(b) with instantiation capabilities

Fig. 18: Example of active stateful PCE operations

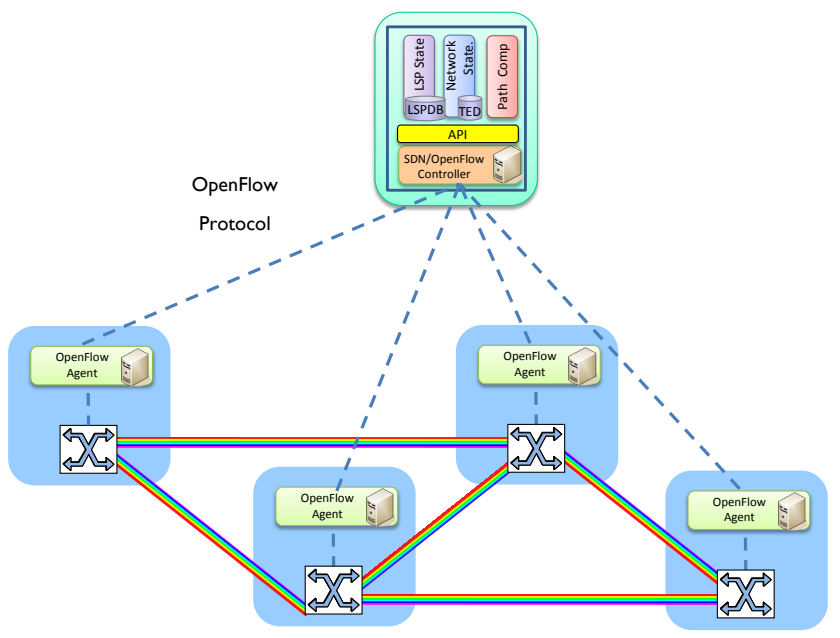

Fig. 19: Example of SDN/OpenFlow-controlled optical network

\section{REFERENCES}

[1] E. Mannie Ed., "Generalized Multi-Protocol Label Switching (GMPLS) Architecture," IETF RFC 3945, October 2004.

[2] R. Muñoz, R. Martínez, R. Casellas et al., "Challenges for GMPLS lightpath provisioning in transparent optical networks: wavelength constraints in routing and signaling," IEEE Communications Magazine, vol. 47, no. 8, pp. 26-34, 2009.

[3] L. Berger Ed., "Generalized multi-protocol label switching (gmpls) resource reservation protocol-traffic engineering (rsvp-te) extensions," IETF RFC 3473, January 2003.

[4] K. Kompella and Y. Rekhter, "Ospf extensions in support of generalized multi-protocol label switching gmpls," IETF RFC 4203, October 2005.

[5] J. Lang Ed., "Link Management Protocol (LMP)," IETF RFC4204, Oct. 2005.

[6] A. Farrel, J.-P. Vasseur, and J. Ash, "A Path Computation Element (PCE)-Based Architecture,” IETF RFC4655, Aug. 2006.

[7] C. Margaria, O. Gonzalez de Dios, and F. Zhang, "Pcep extensions for gmpls," October 2012.
[8] JP. Vasseur Ed. and JL. Le Roux Ed., "Path computation element (pce) communication protocol (pcep)," IETF RFC 5440, March 2009.

[9] Y. Lee Ed. et al., "Path computation element communication protocol (pcep) requirements and protocol extensions in support of global concurrent optimization,” IETF RFC 5557, July 2009.

[10] R. Casellas, R. Muñoz, J. M. Fàbrega, M. S. Moreolo, R. Martínez, L. Liu, T. Tsuritani, and I. Morita, "Gmpls/pce control of flexi-grid dwdm optical networks using co-ofdm transmission [invited]," Journal of Optical Communications and Networking, vol. 4, no. 11, pp. B1-B10, 2012.

[11] R. Casellas, R. Martínez, R. Muñoz, and S. Gunreben, "Enhanced backwards recursive path computation for multi-area wavelength switched optical networks under wavelength continuity constraint," Journal of Optical Communications and Networking, vol. 1, no. 2, pp. A180-A193, 2009.

[12] R. Casellas and Y. Lee, "Pcep extension for wson routing and wavelength assignment," March 2013.

[13] R. Martinez et al., "Challenges and requirements for introducing impairment-awareness into the management and control planes of ASON/GMPLS WDM networks," IEEE Commun. Mag., vol. 44, no. 12, pp. 76-85, Dec. 2006.

[14] R. Martínez, R. Casellas, R. Muñoz, and T. Tsuritani, "Experimental translucent-oriented routing for dynamic lightpath provisioning in gmpls-enabled wavelength switched optical networks," Journal of Lightwave Technology, vol. 28, no. 8, pp. 1241-1255, 2010.

[15] T. Tsuritani, M. Miyazawa, S. Kashihara, and T. Otani, "Optical path computation element interworking with network management system for transparent mesh networks," in Optical Fiber communication/National Fiber Optic Engineers Conference, 2008. OFC/NFOEC 2008. Conference on. IEEE, 2008, pp. 1-10.

[16] F. Cugini, N. Sambo, N. Andriolli, A. Giorgetti, L. Valcarenghi, P. Castoldi, E. Le Rouzic, and J. Poirrier, "Enhancing gmpls signaling protocol for encompassing quality of transmission (qot) in all-optical networks," Journal of Lightwave Technology, vol. 26, no. 19, pp. 3318-3328, 2008.

[17] R. Casellas, R. Muñoz, J. M. Fabrega, M. S. Moreolo, R. Martinez, L. Liu, T. Tsuritani, and I. Morita, "Design and experimental validation of a gmpls/pce control plane for elastic co-ofdm optical networks," Selected Areas in Communications, IEEE Journal on, vol. 31, no. 1, pp. 49-61, 2013.

[18] J. Vasseur, R. Zhang, N. Bitar, and J. Le Roux, "Rfc 5441: A backwardrecursive pce-based computation (brpc) procedure to compute shortest constrained inter-domain traffic engineering label switched paths," IETF, April, 2009.

[19] Q. Zhang, M. M. Hasan, X. Wang, P. Palacharla, and M. Sekiya, "Effi- 


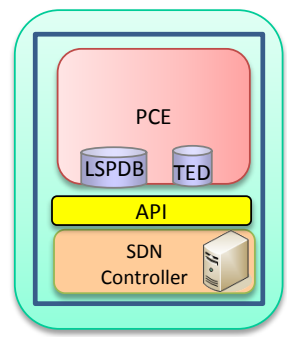

(a) Application

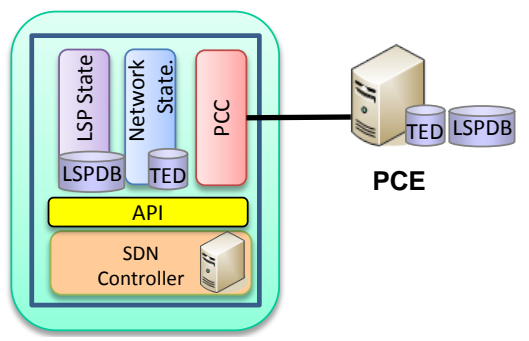

(b) External

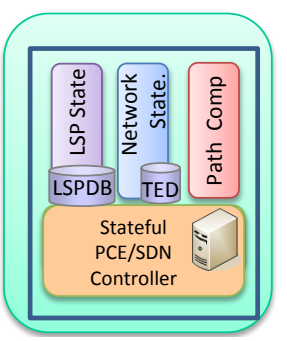

(c) Integrated

Fig. 20: Different models for the integration of a PCE and an OpenFlow controller

cient pce-based survivable path computation in multi-domain networks," in Computer Communications Workshops (INFOCOM WKSHPS), 2011 IEEE Conference on. IEEE, 2011, pp. 139-144.

[20] Zhao, Quintin and Casellas, Ramon and Zhang, Fatai and Farrel, Adrian and Dios, Oscar de, "Extensions to Path Computation Element Communication Protocol (PCEP) for Hierarchical Path Computation Elements (PCE)," IETF draft-zhang-pce-hierarchy-extensions (work in progress), February 2013.

[21] R. Casellas, R. Martínez, R. Muñoz, L. Liu, T. Tsuritani, I. Morita, and M. Tsurusawa, "Dynamic virtual link mesh topology aggregation in multi-domain translucent wson with hierarchical-pce," in European Conference and Exposition on Optical Communications. Optical Society of America, 2011.

[22] R. Martinez, R. Casellas, and R. Muñoz, "Experimental validation/evaluation of a gmpls unified control plane in multi-layer (mplstp/wson) networks," in National Fiber Optic Engineers Conference. Optical Society of America, 2012.

[23] K. Shiomoto, D. Brungard, D. Papadimitriou, and M. Vigoureux, "Rfc 5212: Requirements for gmpls-based multi-region and multi-layer networks (mrn/mln)," IETF, july, 2008.

[24] R. Muñoz, R. Vilalta, R. Casellas, R. Martínez, and F. J. Vílchez, "First lab trial of an integrated mpls-tp/wson transport network controlled by a unified gmpls control plane in the adrenaline testbed," in Photonics in Switching (PS), 2012.

[25] R. Casellas, R. Martínez, R. Muñoz, L. Liu, T. Tsuritani, and I. Morita, "Inter-layer traffic engineering with hierarchical-pce in mpls-tp over wavelength switched optical networks," in European Conference and Exhibition on Optical Communication. Optical Society of America, 2012.

[26] G. Swallow, H. Ishimatsu, Y. Rekhter, and J. Drake, "Generalized multiprotocol label switching (gmpls) user-network interface (uni): resource reservation protocol-traffic engineering (rsvp-te) support for the overlay model," IETF, October, 2005.

[27] Q. Zhao, R. Casellas, F. Zhang, A. Farrel, and O. d. Dios, "Extensions to path computation element communication protocol (pcep) for hierarchical path computation elements (pce)," July 2012.

[28] J. Medved, S. Ray, H. Gredler, A. Farrel, and S. Previdi, "North-bound distribution of link-state and te information using bgp," May 2013.

[29] O. G. de Dios, F. J. Chico, and F. M. del Nuevo, "Benefits of limited context awareness in stateless pce," in Optical Fiber Communication Conference and Exposition (OFC/NFOEC), 2011 and the National Fiber Optic Engineers Conference. IEEE, 2011, pp. 1-3.

[30] A. Giorgetti, F. Cugini, N. Sambo, F. Paolucci, N. Andriolli, and P. Castoldi, "Path state-based update of pce traffic engineering database in wavelength switched optical networks," Communications Letters, IEEE, vol. 14, no. 6, pp. 575-577, 2010.

[31] R. Martínez, A. Castro, R. Casellas, R. Muñoz, L. Velasco, R. Vilalta, and J. Comellas, "Experimental validation of dynamic restoration in gmpls-controlled multi-layer networks using pce-based global concurrent optimization," in Optical Fiber Communication Conference. Optical Society of America, 2013.

[32] J. Medved, E. Crabbe, I. Minei, and R. Varga, "Pcep extensions for stateful pce," May 2013.

[33] R. Casellas, R. Martínez, R. Muñoz, L. Liu, T. Tsuritani, and I. Morita, "Dynamic provisioning via a stateful pce with instantiation capabilities in gmpls-controlled flexi-grid dwdm networks," in European Conference and Exhibition on Optical Communication, 2013.

[34] E. Crabbe, S. Sivabalan, I. Minei, and R. Varga, "Pcep extensions for pce-initiated lsp setup in a stateful pce model," April 2013.
[35] N. McKeown, T. Anderson, H. Balakrishnan, G. Parulkar, L. Peterson, J. Rexford, S. Shenker, and J. Turner, "Openflow: enabling innovation in campus networks," ACM SIGCOMM Computer Communication Review, vol. 38, no. 2, pp. 69-74, 2008.

[36] M. Channegowda, R. Nejabati, M. Rashidi Fard, S. Peng, N. Amaya, G. Zervas, D. Simeonidou, R. Vilalta, R. Casellas, R. Martínez et al., "Experimental demonstration of an openflow based software-defined optical network employing packet, fixed and flexible dwdm grid technologies on an international multi-domain testbed," Optics Express, vol. 21, no. 5, pp. 5487-5498, 2013.

[37] V. R. Gudla, S. Das, A. Shastri, G. Parulkar, N. McKeown, L. Kazovsky, and S. Yamashita, "Experimental demonstration of openflow control of packet and circuit switches," in Optical Fiber Communication Conference. Optical Society of America, 2010.

[38] L. Liu, T. Tsuritani, I. Morita, H. Guo, and J. Wu, "Openflow-based wavelength path control in transparent optical networks: a proof-ofconcept demonstration," in Optical Communication (ECOC), 2011 37th European Conference and Exhibition on, 2011, p. Tu.5.K.2.

[39] L. Liu, R. Muñoz, R. Casellas, T. Tsuritani, R. Martínez, and I. Morita, "Openslice: an openflow-based control plane for spectrum sliced elastic optical path networks," Optics express, vol. 21, no. 4, pp. 4194-4204, 2013.

[40] - "Openslice: an openflow-based control plane for spectrum sliced elastic optical path networks," in European Conference and Exhibition on Optical Communication, 2012, p. Mo.2.D.3.

[41] S. Das, "Extensions to the openflow protocol in support of circuit switching," Addendum to OpenFlow protocol specification (v1.0)Circuit Switch Addendum v0, vol. 3, 2010.

[42] R. Casellas, R. Martínez, R. Muñoz, R. Vilalta, L. Liu, T. Tsuritani, and I. Morita, "Control and management of flexi-grid optical networks with an integrated stateful pce and openflow controller," Accepted for publication in Journal of Optical Communications and Networking, 2013.

[43] L. Liu, R. Casellas, T. Tsuritani, I. Morita, R. Martínez, and R. Muñoz, "Experimental demonstration of an openflow/pce integrated control plane for ip over translucent wson with the assistance of a per-requestbased dynamic topology server," in European Conference and Exhibition on Optical Communication. Optical Society of America, 2012.

[44] R. Casellas, R. Martínez, R. Muñoz, L. Liu, T. Tsuritani, and I. Morita, "An integrated stateful pce/openflow controller for the control and management of flexi-grid optical networks," in Optical Fiber Communication Conference. Optical Society of America, 2013. 
Raül Munoz (SM'12) graduated in telecommunications engineering in 2001 and received a Ph.D. degree in telecommunications in 2005 , both from the Universitat Politècnica de Catalunya (UPC), \begin{tabular}{l|l} 
PLACE & Spain. After working as undergraduate researcher at
\end{tabular} PHOTO Telecom Italia Lab (Turin, Italy) in 2000, and as HERE $\quad$ assistant professor at the UPC in 2001, he joined the Centre Tecnològic de Telecomunicacions de Catalunya (CTTC) in 2002. Currently, he is Senior Researcher at CTTC. Since 2000, he has participated in several R\&D projects funded by the European Commission's Framework Programmes (FP7, FP6 and FP5) and the Spanish Ministries, as well as technology transfer projects. He leads the European Consortium of the EU-Japan project STRAUSS, and the Spanish project FARO. His research interests include control and service management architectures, protocols and traffic engineering algorithms for future optical networks.

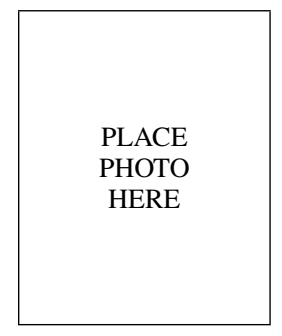

Ramon Casellas (SM'12) graduated in telecommunications engineering in 1999 by both the UPCBarcelonaTech university and ENST Telecom Paristech, within an Erasmus/Socrates double degree program. After working as an undergraduate researcher at both France Telecom R\&D and British Telecom Labs, he completed a Ph.D. degree in 2002. He worked as an associate professor at the networks and computer science deptartment of the ENST (Paris) and joined the CTTC Optical Networking Area in 2006, with a Torres Quevedo research grant. He currently is a research associate and the coordinator of the ADRENALINE testbed. He has been involved in several international R\&D and technology transfer projects. His research interests include the GMPLS/PCE architecture and protocols, software defined networking and traffic engineering mechanisms. He contributes to IETF standardization within the CCAMP and PCE working groups. He is a member of the IEEE Communications Society and a member of the Internet Society.

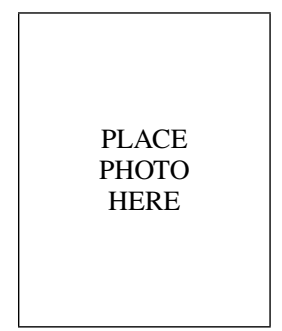

Ricardo Martínez graduated in telecommunications engineering 2002 and received a Ph.D. degree in telecommunications in 2007, both from the Technical University of Catalonia, Spain. Since June 2007 he has been a research associate in the CTTC Optical Networking Area. He has participated in several Spanish, EU-funded (EU FP6, FP7 and CELTIC) and Industrial projects in the context of optical networking. His research interests include GMPLS architecture, distributed control schemes, packet and optical transport networks. He has published over 80 scientific conferences and peer reviewed journal papers.

\begin{tabular}{|c|} 
\\
\\
PLACE \\
PHOTO \\
HERE \\
\end{tabular}

Ricard Vilalta graduated in Telecommunications Engineering in 2007. Ricard Vilalta worked as research engineer and software developer in Triagnosys GmbH (Munich, Germany) during 2006-2009, leading projects on satellite networks. In 2010, Ricard Vilalta joined CTTC as Research Engineer at the Optical Networking Area. Since 2010, Ricard Vilalta is Ph.D. Candidate in Signal Theory and Communications Department at Technical University of Catalonia (UPC). 